\title{
Avaliação de carga de trabalho em alunos de pós-graduação em engenharia de produção: um estudo exploratório
}

\section{Assessment of the workload of industrial engineering graduate students: an exploratory study}

\author{
Tatiana Cecagno Galvan \\ Gabriela Musse Branco ${ }^{1}$ \\ Tarcísio Abreu Saurin ${ }^{1}$
}

\begin{abstract}
Resumo: Os resultados educacionais estão ligados à carga de trabalho do estudante que, por sua vez, sofre influência de fatores individuais e do contexto institucional. Contudo, no âmbito da pós-graduação brasileira, e mais especificamente no caso da Engenharia de Produção, pouco é conhecido sobre a intensidade dessa carga de trabalho, fatores contribuintes e meios de gerenciá-la. Este estudo tem como objetivo realizar uma avaliação exploratória da carga de trabalho em estudantes de Pós-Graduação em Engenharia de Produção. A avaliação foi realizada em um curso nacional de excelência na área, por meio de: entrevistas semiestruturadas com três alunos, aplicação de um questionário (NASA-TLX adaptado) respondido por trinta e três alunos; reunião de feedback dos resultados dos questionários aos alunos. Os dados indicaram que a carga de trabalho é alta e similar entre as diferentes modalidades do curso: mestrado profissional, mestrado acadêmico e doutorado. Além disso, foi possível identificar o alto nível de frustração entre alunos do mestrado profissional, o alto nível de esforço no mestrado acadêmico, a grande demanda mental do doutorado, e a maior demanda temporal para quem exerce outras atividades profissionais além dos estudos. Dentre os fatores contribuintes nesta alta carga de trabalho, foi identificada a falta de acompanhamento do orientador, a realização de trabalhos acadêmicos não diretamente ligados ao projeto de pesquisa, a alta exigência imposta pelos estudantes a si mesmos e o medo de não finalizar o trabalho.
\end{abstract}

Palavras-chave: Carga de trabalho; Pós-graduação; NASA-TLX; Engenharia de produção; Aprendizagem.

\begin{abstract}
Academic performance depends on the workload of students, and it is influenced both by individual factors and the institutional context. However, in the Brazilian post-graduation, more specifically in the area of industrial engineering, little is known about the workload intensity, contributing factors, and control measures. This study presents an exploratory assessment of the workload of graduate students of industrial engineering. The assessment was carried out in a leading national post-graduation program. It was based on semi-structured interviews with three students; the application of a questionnaire (adapted from the NASA-TLX) to thirty-three students; and a feedback meeting to discuss the results of the questionnaire. The results indicated that the workload is very high and similar across the three levels of post-graduation: professional master's degree, academic master's degree, and PhD. Other noteworthy results were related to the high level of frustration of the students of professional master's degrees; the high level of effort in the academic master's degrees; the high mental demand in the PhDs; and the high temporal demand for students who have professional activities in addition to the academic ones. Some contributing factors for the high workload were also identified, such as insufficient support from the supervisor; involvement with academic activities that are not directly related to the research project; self-imposed pressure for high performance; and fear of not meeting the deadline.
\end{abstract}

Keywords: Workload; Post-graduation; NASA-TLX; Industrial engineering; Learning.

\footnotetext{
${ }^{1}$ Programa de Pós-Graduação em Engenharia de Produção, Departamento de Engenharia de Produção, Universidade Federal do Rio Grande do Sul - UFRGS, Av. Osvaldo Aranha, 99, $5^{\circ}$ Andar, Bairro Bom Fim, CEP 90035-190, Porto Alegre, RS, Brasil, e-mail: tatiana@producao.ufrgs.br; gabriela.branco@proplan.ufrgs.br; saurin@ufrgs.br
}

Recebido em Jun. 2, 2014 - Aceito em Maio 5, 2015

Suporte financeiro: este artigo recebeu suporte financeiro no formato de bolsa CNPQ de Mestrado, referente à autora Tatiana Cecagno Galvan. 


\section{Introdução}

Há uma crescente mudança no Brasil no que se refere ao acesso à educação por parcelas maiores da população. Segundo o IBGE (2004, 2012), no período de 2000 a 2010 houve um aumento de aproximadamente $300 \%$ no acesso ao mestrado e doutorado, na faixa etária de 25 a 29 anos de idade. Nesse universo de estudantes, muitos combinam seus estudos com compromissos profissionais e familiares, em que cada papel exige obrigações por vezes conflitantes, o que pode resultar em aumento da carga de trabalho (CT) (Adebayo, 2006) e em dificuldades na aprendizagem (Darmody et al., 2008).

Outro fator que pode contribuir para o aumento da carga de trabalho é o pouco tempo fornecido pelos professores para a realização das atividades. Isso pode levar os alunos a perceberem que o tempo para completar a tarefa é inatingível, contribuindo para a aprendizagem de superfície. O mesmo tipo de aprendizagem ocorre quando os alunos percebem que há excesso de avaliações (Lewis \& Webb, 2009). Aprendizagem de superfície refere-se à falta de postura crítica e interesse sincero na aprendizagem. Esse tipo se contrapõe à aprendizagem profunda, na qual o aluno tem um interesse sincero na tarefa e concentra-se na compreensão do significado subjacente. Tal tipo de aprendizagem requer que o aluno tenha tempo para completar as tarefas acadêmicas (Kyndt et al., 2011; Lewis \& Webb, 2009).

O aumento da carga de trabalho tem efeito nos resultados educacionais, podendo levar à ausência nas aulas e diminuição no desempenho das avaliações (Darmody et al., 2008). A alta carga de trabalho também está associada à baixa produtividade, baixa satisfação no trabalho, síndrome de burnout e intenção de deixar os estudos. Há também implicações negativas para a saúde, tais como ansiedade, depressão, infarto do miocárdio, abuso de drogas, absenteísmo e assédio moral (Panari et al., 2012). De outro lado, vale ressaltar que a carga de trabalho insuficiente também pode ser prejudicial, levando a algumas consequências similares às do excesso de carga de trabalho, como a insatisfação (Gregoriades \& Sutcliffe, 2008).

Estudos em diversos países têm demonstrado preocupação com a carga de trabalho dos estudantes, usando diferentes métodos de avaliação. Em trabalho realizado sobre o estresse ocupacional em estudantes mulheres na Alemanha, Myrtek et al. (1996) verificaram que a carga de trabalho mental cresce conforme avança-se no curso. Os autores inicialmente solicitaram que os sujeitos-alvo da pesquisa respondessem questionários para avaliar características de personalidade, hábitos pessoais, perfil socioeconômico, e nível de estresse. Em uma segunda etapa, a avaliação da carga mental ocorreu por meio da colocação de eletrodos e detectores de movimento nos estudantes.
Jacobs \& Dodd (2003) realizaram estudo sobre nível de burnout em estudantes de psicologia de uma universidade nos EUA, concluindo que a carga de trabalho influencia no desgaste psicológico dos estudantes. Esses autores também aplicaram questionários a grupos de estudantes. Nesse caso, foi usado o Maslach Burnout Inventory (MIB), um questionário com 22 itens divididos em três grupos: exaustão emocional, despersonalização e realização pessoal.

Em estudo realizado com alunos do primeiro ano de doutorado profissional em Farmácia nos Estados Unidos, Geslani \& Gaebelein (2013) identificaram um alto nível de estresse, bem como que a carga mental estava relacionada ao estresse. Essa avaliação ocorreu por meio de questionários baseados na Perceived Stress Scale (PSS10) para determinar o estresse percebido pelos estudantes e no Health-related Quality of Life Scale (HRQOL-14), para avaliação da saúde física e mental.

Neste contexto, o presente estudo apresenta uma avaliação exploratória da carga de trabalho em estudantes de Pós-Graduação em Engenharia de Produção. Tal área vem tendo forte expansão acadêmica na última década no país, em nível de graduação e pós-graduação, o que indica a sua relevância no contexto educacional brasileiro. Também vale ressaltar que, no nível nacional, não foram identificados estudos acerca da intensidade, fatores contribuintes, e ações de controle da carga de trabalho em estudantes de pós-graduação. A principal ferramenta de avaliação de carga de trabalho usada foi o questionário NASA-TLX (Hart \& Staveland, 1988), que ainda não havia sido usado neste contexto.

\section{Referencial teórico}

\subsection{Definição de carga de trabalho adotada neste estudo}

De acordo com Stoll et al. (2007), carga de trabalho é qualquer esforço fisiológico resultante de reações. Há ainda autores que definem a carga de trabalho em função do número de horas trabalhadas (Balch et al., 2010; Johnson \& Smith, 2008). Neste estudo, é utilizada a definição de Hart \& Staveland (1988), que entendem a carga de trabalho como um constructo subjetivo, definido pela percepção do esforço despendido para alcançar um determinado nível de desempenho.

\subsection{Avaliação da carga de trabalho}

As ferramentas para avaliação da carga de trabalho se enquadram nas seguintes categorias: medidas baseadas no desempenho, medidas fisiológicas, e medidas subjetivas (Meshkati et al., 1992). As medidas baseadas no desempenho dizem respeito ao rendimento de uma 
tarefa ou tarefas múltiplas realizadas pelo indivíduo, assumindo que quanto maior a complexidade de uma tarefa, maiores são suas exigências e maior é a carga de trabalho (Cardoso \& Gontijo, 2012). Já as medidas fisiológicas medem as respostas do organismo às mudanças nos níveis da carga de trabalho. Entre as principais medidas fisiológicas, estão as relacionadas aos olhos, coração e cérebro. São exemplos dessas medidas, a frequência de movimento dos olhos, duração das piscadas, frequência cardíaca, variação de ondas cerebrais, potencial muscular e frequência da respiração (Cardoso \& Gontijo, 2012; Gregoriades $\&$ Sutcliffe, 2008).

Quanto às medidas subjetivas, como o nome indica, elas são baseadas em percepções subjetivas decorrentes das experiências relacionadas à carga de trabalho. O método NASA-TLX (National Aeronautics and Space Administration - Task Load Index) enquadra-se nessa categoria, tendo sido desenvolvido pela Aerospace Human Factors Research Division da NASA-Ames Research Center e publicado pela primeria vez por Hart \& Staveland (1988). O NASA-TLX adaptado (Diniz, 2003) utiliza seis fatores para definir um valor multidimensional da carga de trabalho geral percebida pela população pesquisada. As seguintes dimensões são consideradas:

- Demanda física: atividade física para a realização do trabalho.

- Demanda mental: atividade mental requerida para a realização do trabalho (por exemplo, tomada de decisões).

- Demanda temporal: nível de pressão imposto para a realização do trabalho.

- Esforço físico e mental: o quanto é necessário trabalhar física e mentalmente para atingir um nível desejado de desempenho.

- Desempenho: nível de satisfação com o desempenho pessoal para realização do trabalho. Avalia o quanto de satisfação foi obtida com o desempenho para alcançar o desfecho final.

- Nível de frustação: o quanto se é irritado, desencorajado, contrariado, inseguro versus satisfeito, motivado, relaxado e seguro durante a realização da tarefa.

$\mathrm{Na}$ aplicação desse método, inicialmente o respondente realiza uma comparação entre os fatores, escolhendo entre cada par de fatores qual é o mais influente na sua carga de trabalho. Assim, são feitas quinze comparações entre pares. $\mathrm{O}$ segundo passo requer que o pesquisado assinale o nível de influência ou contribuição de cada um dos fatores para a carga de trabalho.

\subsection{Carga de trabalho em estudantes de pós-graduação}

Estudantes de Pós-Graduação são confrontados com várias fontes de estresse, dentre as quais podem ser citadas um conflito de papéis (profissional graduado e estudante) e sobreposição de funções acadêmicas e não acadêmicas. A dificuldade de gerir as finanças é outra fonte de estresse frequente, pois o tempo despendido com os estudos limita a possibilidade de exercer atividades remuneradas. O baixo poder de decisão em relação a aspectos essenciais da sua experiência estudantil, como orientador e temas de pesquisa, também podem levar ao estresse (Grady et al., 2014; Erichsen \& Bolliger, 2011; Adamo, 2013). De acordo com Graybill et al. (2006), quanto maior a natureza multidisciplinar do curso, maior tende a ser a carga de trabalho dos estudantes, pois o aluno deve compreender paradigmas científicos que aparentemente podem ser divergentes, necessitando de maior tempo para tal compreensão, sob pena de desorientação intelectual. A ausência de feedback positivo e o isolamento social são apontados como fatores contribuintes para a carga de trabalho por Grady et al. (2014) e Erichsen \& Bolliger (2011).

Outro aspecto importante na pós-graduação é a relação entre professor e aluno, especialmente na orientação da dissertação ou tese. Estudante e professor trabalham sob a pressão de sua carga de trabalho, outros deveres e responsabilidades. No entanto, esta relação por vezes provoca sofrimento interpessoal, devido à falta de supervisão, às diferenças de expectativas entre orientador e orientando e falhas na comunicação (Erichsen \& Bolliger, 2011). Portanto, nesse cenário, é comum encontrar estudantes com uma sensação de muitas atividades para fazer, mas com dificuldade de priorizá-las e cumprir metas individuais e institucionais (Grady et al., 2014; Erichsen \& Bolliger, 2011).

\section{Método de pesquisa}

\subsection{Delineamento da pesquisa}

A avaliação da carga de trabalho dos alunos de pós-graduação foi conduzida em três etapas: planejamento da aplicação do questionário e das entrevistas; coleta de dados e análise dos dados. O público-alvo da pesquisa foram estudantes de pós-graduação nos níveis de mestrado (acadêmico e profissional) e doutorado do curso de Engenharia de Produção da Universidade Federal do Rio Grande do Sul (UFRGS), curso existente há mais de 20 anos, que, recentemente, (2013) obteve conceito 6 na avaliação da CAPES. A escolha da população considerou a facilidade de acesso dos pesquisadores e definiu-se que seriam coletados no mínimo 30 questionários. 


\subsection{Planejamento e coleta de dados}

Nessa pesquisa, foi utilizado o questionário NASA-TLX adaptado por Diniz (2003). O questionário continha uma folha de rosto para identificação do sujeito pesquisado, com os seguintes campos: nome (opcional); idade; sexo; modalidade (mestrado acadêmico, mestrado profissional ou doutorado); curso, pois, se o aluno fosse de outro curso e estivesse apenas cursando disciplinas da Engenharia de Produção, as respostas não seriam analisadas; data de início do curso; e a pergunta "você trabalha fora do mestrado/doutorado?" com opções de "sim" ou "não". Em seguida, eram apresentados os conceitos utilizados no instrumento em um glossário, como citado no item 2.2 deste estudo. Havia um formulário para comparação aos pares dos seis fatores, no qual o respondente deveria assinalar qual fator era mais influente para a carga de trabalho. Por último, pedia-se para o respondente assinalar a influência de cada fator em uma escala de $15 \mathrm{~cm}$ (Anexo 1).

Os questionários foram enviados por meio eletrônico a todos os alunos vinculados ao curso de pós-graduação da Engenharia de Produção da UFRGS, totalizando o envio para 174 alunos, sendo 63 do mestrado acadêmico, 50 do mestrado profissional e 61 do doutorado. No convite, foram explicados os objetivos do trabalho e as instruções de como preencher e retornar o questionário. A coleta foi realizada no período de 15 dias, no segundo mês do segundo semestre letivo de 2013. Ao final do período, obtiveram-se 33 questionários válidos (19\%), sendo considerado válido o questionário completamente respondido, exceto o nome do indivíduo, que era uma resposta opcional.

Além do questionário, foram realizadas entrevistas com um representante de cada público-alvo pesquisado, ou seja, um doutorando, um mestrando acadêmico e um mestrando profissional, escolhidos por conveniência. Foi agendada uma data específica para as entrevistas, às quais todos os entrevistados compareceram, com hora marcada. Tais entrevistas iniciaram-se com a seguinte pergunta: "quais suas maiores dificuldades relacionadas à pós-graduação?" Dois entrevistados eram do sexo feminino e um do sexo masculino, com idades entre 25 e 29 anos. As entrevistas duraram, em média, 20 minutos, foram gravadas e transcritas.

\subsection{Análise dos dados}

Para a análise dos resultados dos questionários, a Equação 1 foi utilizada para o cálculo da carga de trabalho de cada aluno:

$$
C T_{n}=\frac{\sum_{i=1}^{6}(F i) \cdot(P F i)}{15}
$$

em que:

- CTn: Carga de Trabalho do indivíduo $\mathrm{n}(1 \geq \mathrm{n} \geq 33)$;
- Fi: contagem atribuída a cada fator na comparação entre eles;

- PFi: peso atribuído à influência de cada fator.

Conforme recomendado por Holsbach (2005), o nível de carga de trabalho foi classificado em três faixas: (a) 5 a 7, médio risco; (b) 7,1 a 11, alto risco, (c) 11,01 a 15, máximo risco.

Quanto às entrevistas, depois de transcritas, elas foram analisadas visando à identificação de trechos que apontassem fatores que contribuem para a maior ou menor carga de trabalho, assim como trechos que indicassem as diferentes dimensões da carga de trabalho.

Além disso, foi realizada uma análise individual dos fatores da pesquisa. Para tanto, multiplicou-se a contagem dos fatores (a partir da comparação aos pares) pela sua influência e dividiu-se esse resultado por quinze, obtendo-se, assim, um valor entre zero (0) e cinco (5) para cada fator de cada entrevistado, conforme Equação 2. Tendo-se os resultados de cada fator para cada indivíduo entrevistado, fez-se a média dos fatores para a amostra. Quanto mais alto o valor do fator, maior a sua influência na carga de trabalho.

$$
V F_{i n}=\frac{F i \cdot P F i}{15}
$$

em que:

- $V F n$ : Valor do Fator i ( $1 \geq \mathrm{i} \geq 6)$ para o indivíduo $\mathrm{n}(1 \geq \mathrm{n} \geq 33)$;

- Fi: contagem atribuída ao fator na comparação entre eles;

- PFi: peso atribuído à influência do fator.

\subsection{Reunião de feedback dos resultados}

Os resultados do questionário foram apresentados a alguns dos respondentes (três doutorandos, um mestrando profissional e 6 mestrandos acadêmicos), que demonstraram interesse nesses dados. Antes do início da apresentação, visando estimular o debate e obter dados adicionais para interpretar os resultados do questionário, duas questões foram propostas ao grupo: Como está sua carga de trabalho?; e O que prejudica sua carga de trabalho? Em seguida, os resultados do questionário foram apresentados e os participantes foram convidados a apontar possíveis explicações. A reunião durou cerca de 30 minutos.

\section{Resultados e discussões}

\subsection{Caracterização da amostra}

Dentre os 33 questionários válidos, nove foram respondidos por alunos de doutorado $(27,27 \%)$, 17 de mestrado acadêmico (51,52\%) e 7 de mestrado 
profissional $(21,21 \%)$, conforme Tabela 1. Em relação ao sexo dos respondentes, foram 16 mulheres $(48,48 \%)$ e 17 homens $(51,52 \%)$. Aproximadamente $40 \%$ do total de respondentes válidos estavam com mais da metade do tempo do curso concluído e, portanto, tinham um conhecimento maior acerca das demandas do curso. Do mesmo modo, 21 alunos trabalham fora da pós-graduação $(63,64 \%)$ enquanto $12(36,36 \%)$ não exercem atividade profissional além da pós. Também vale salientar que, durante o período de créditos, os alunos das três modalidades têm aulas apenas no turno diurno. Não foram coletados dados acerca dos horários de estudos extraclasse.

\subsection{Resultados dos questionários e entrevistas}

A Tabela 2 apresenta os resultados, para cada respondente, segundo a comparação entre os seis fatores (totalizando 15 comparações). Os valores nas células da tabela variam de 0 a 5 , sendo que 0 indica que aquele fator não foi priorizado em relação a nenhum outro e 5 indica que ele foi priorizado em relação a cada um dos outros. Assim, quanto maior o número de vezes que o fator foi priorizado, maior sua relevância.

A Tabela 3 apresenta os resultados da influência percebida de cada fator, segundo a escala de 0 (nenhuma influência) a 15 (grande influência).

A partir das Tabelas 2 e 3 foi possível obter a carga de trabalho individual de cada estudante. Para tanto, foi realizada a soma da multiplicação da contagem de cada fator pela sua influência, dividindo-se esse resultado por 15, conforme a Equação 1 . O resultado geral da carga de trabalho da amostra pode ser obtido pela média dos resultados individuais. A Tabela 4 apresenta as cargas de trabalho individuais e a Tabela 5, as cargas por categoria.

Conforme classificação sugerida por Holsbach (2005), os resultados da Tabela 4 indicam carga de trabalho de máximo risco, para todas as categorias. $O$ relato do mestrando acadêmico entrevistado ilustra essa situação:

[...] se a cobrança continuar neste ritmo, através de avaliações por artigos para cada disciplina, mais atividades domiciliares diárias, serei obrigado a abdicar do programa de pós-graduação ou da minha vida pessoal [...].

Tabela 1. Questionários enviados e válidos.

\begin{tabular}{ccc}
\hline Categoria & $\begin{array}{c}\text { Questionários } \\
\text { enviados }\end{array}$ & $\begin{array}{c}\text { Questionários } \\
\text { válidos }\end{array}$ \\
\hline Doutorado & 61 & 9 \\
Mestrado & 63 & 17 \\
Acadêmico & & 7 \\
Mestrado & 50 & \\
Profissional & & \\
\hline
\end{tabular}

De outro lado, embora os resultados conforme o sexo sejam similares, outros estudos, como o de Al-Sowygh (2013), concluíram que estudantes do sexo feminino percebem maior carga que os do sexo masculino. Os resultados também foram muito similares entre os alunos que trabalham fora e os que não trabalham, embora Adebayo (2006) tenha concluído que a demanda de trabalho pode dificultar os estudos e a demanda proveniente desses pode dificultar o desempenho profissional.

Em relação à análise individual dos fatores, a Figura 1 apresenta as médias gerais. Já as Figuras 2 e 3

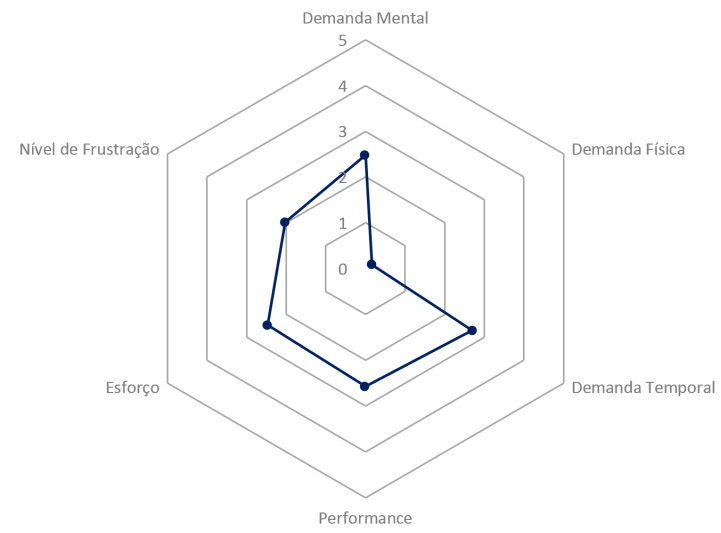

Figura 1. Média geral dos fatores.

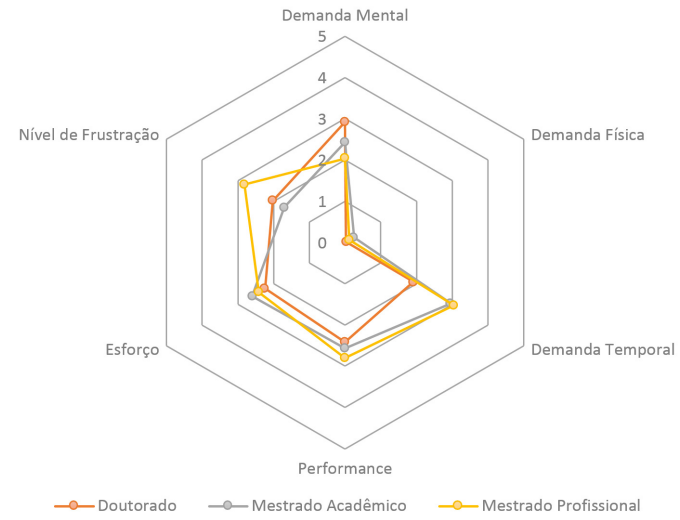

Figura 2. Média dos fatores por tipo de curso.

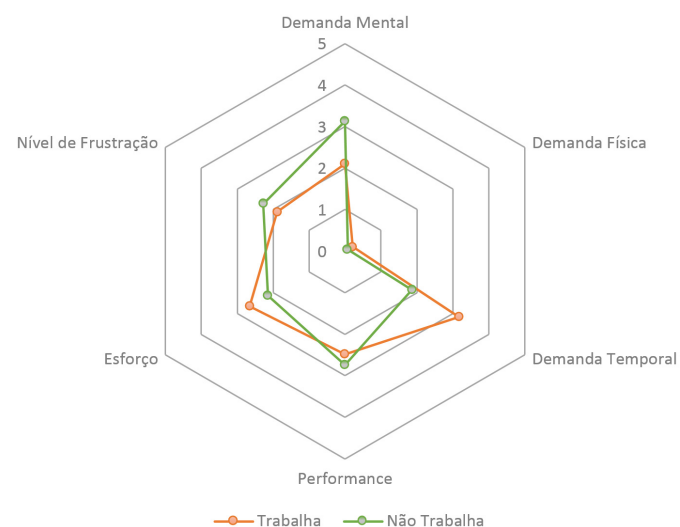

Figura 3. Média dos fatores por alunos que trabalham ou não. 
Tabela 2. Resultados da comparação entre os pares de fatores que têm impacto na carga de trabalho.

\begin{tabular}{|c|c|c|c|c|c|c|c|c|c|}
\hline 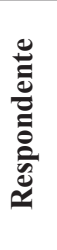 & 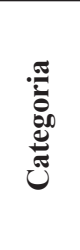 & $\begin{array}{l}\stackrel{\theta}{\ddot{D}} \\
\ddot{D}\end{array}$ & 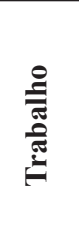 & 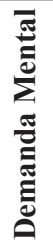 & 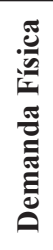 & 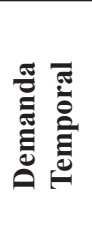 & 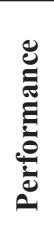 & 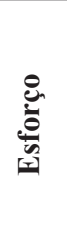 & 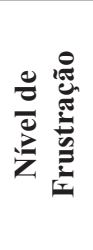 \\
\hline 1 & $\mathrm{D}$ & $\mathrm{F}$ & Não & 3 & 0 & 1 & 2 & 4 & 5 \\
\hline 2 & $\mathrm{D}$ & $\mathrm{M}$ & Não & 5 & 0 & 3 & 4 & 2 & 1 \\
\hline 3 & D & $\mathrm{M}$ & Sim & 4 & 0 & 4 & 1 & 4 & 2 \\
\hline 4 & D & $\mathrm{F}$ & Sim & 4 & 0 & 4 & 3 & 2 & 2 \\
\hline 5 & $\mathrm{D}$ & $\mathrm{M}$ & Sim & 4 & 0 & 3 & 5 & 2 & 1 \\
\hline 6 & $\mathrm{D}$ & $\mathrm{F}$ & Sim & 2 & 0 & 1 & 3 & 4 & 5 \\
\hline 7 & $\mathrm{D}$ & $\mathrm{M}$ & Não & 3 & 0 & 1 & 4 & 4 & 3 \\
\hline 8 & $\mathrm{D}$ & $\mathrm{F}$ & Sim & 4 & 1 & 2 & 3 & 5 & 0 \\
\hline 9 & $\mathrm{D}$ & M & Sim & 2 & 0 & 3 & 3 & 2 & 5 \\
\hline 10 & MA & $\mathrm{M}$ & Não & 1 & 0 & 2 & 4 & 3 & 5 \\
\hline 11 & MA & $\mathrm{M}$ & Sim & 2 & 0 & 5 & 4 & 3 & 1 \\
\hline 12 & MA & $\mathrm{F}$ & Não & 3 & 0 & 2 & 5 & 4 & 1 \\
\hline 13 & MA & $\mathrm{F}$ & Não & 2 & 0 & 3 & 1 & 5 & 4 \\
\hline 14 & MA & $\mathrm{M}$ & Sim & 2 & 3 & 5 & 2 & 3 & 0 \\
\hline 15 & MA & $\mathrm{M}$ & Sim & 2 & 0 & 4 & 1 & 5 & 3 \\
\hline 16 & MA & $\mathrm{F}$ & Sim & 1 & 0 & 5 & 4 & 3 & 2 \\
\hline 17 & MA & $\mathrm{M}$ & Não & 3 & 0 & 5 & 1 & 2 & 4 \\
\hline 18 & MA & $\mathrm{F}$ & Não & 5 & 0 & 1 & 4 & 2 & 3 \\
\hline 19 & MA & $\mathrm{F}$ & Sim & 3 & 1 & 4 & 2 & 5 & 0 \\
\hline 20 & MA & $\mathrm{F}$ & Sim & 2 & 0 & 5 & 1 & 3 & 4 \\
\hline 21 & MA & $\mathrm{F}$ & Não & 4 & 0 & 1 & 5 & 3 & 2 \\
\hline 22 & MA & $\mathrm{M}$ & Não & 4 & 1 & 5 & 1 & 2 & 2 \\
\hline 23 & MA & $\mathrm{M}$ & Sim & 2 & 0 & 1 & 4 & 5 & 3 \\
\hline 24 & MA & $\mathrm{M}$ & Sim & 2 & 0 & 5 & 4 & 3 & 1 \\
\hline 25 & MA & $\mathrm{F}$ & Não & 3 & 0 & 1 & 5 & 3 & 3 \\
\hline 26 & MA & M & Não & 5 & 0 & 3 & 4 & 2 & 1 \\
\hline 27 & MP & $\mathrm{F}$ & Sim & 2 & 0 & 2 & 3 & 3 & 5 \\
\hline 28 & MP & $\mathrm{F}$ & Sim & 1 & 0 & 4 & 4 & 3 & 3 \\
\hline 29 & $\mathrm{MP}$ & $\mathrm{F}$ & Sim & 4 & 1 & 5 & 3 & 2 & 0 \\
\hline 30 & MP & $\mathrm{M}$ & Sim & 3 & 0 & 1 & 5 & 4 & 2 \\
\hline 31 & MP & $\mathrm{F}$ & Sim & 2 & 0 & 1 & 4 & 3 & 5 \\
\hline 32 & MP & $\mathrm{M}$ & Sim & 1 & 0 & 5 & 2 & 3 & 4 \\
\hline 33 & MP & $\mathrm{M}$ & Sim & 3 & 0 & 5 & 1 & 2 & 4 \\
\hline
\end{tabular}

apresentam os resultados por categoria de pós e alunos que trabalham ou não, respectivamente. Pela Figura 1 é possível perceber que os alunos não percebem demanda física, sendo muito baixa por unanimidade. Já a demanda temporal, o esforço e o desempenho obtiveram um grau maior de percepção. Essa diferença confirma as características de um curso de pós-graduação que não apresenta trabalho físico, concentrando sua demanda em atividades que exigem tempo, esforço e demanda mental, tais como trabalhos de aula, provas, dissertação, pesquisa.

Ao se compararem os diferentes níveis de pós-graduação, nota-se que o nível de frustração do mestrado profissional é consideravelmente maior que o dos demais e que, no doutorado, a demanda mental é maior, embora a demanda temporal tenha sido menor que em ambas as categorias do mestrado. O nível de frustração do mestrado profissional pode estar relacionado às características acadêmicas do curso, não correspondendo às expectativas dos alunos para algo mais aplicado à sua vida profissional. A maior demanda mental do doutorado justifica-se pela exigência de desenvolvimento teórico mais aprofundado do que os outros cursos, enquanto que a menor demanda temporal está relacionada ao maior tempo disponível para conclusão do curso (4 anos) em relação aos outros (2 anos). Já em relação às diferentes percepções dos alunos que trabalham dos 
Tabela 3. Influência dos fatores.

\begin{tabular}{|c|c|c|c|c|c|c|c|}
\hline Respondente & $\begin{array}{c}\text { Demanda } \\
\text { Mental }\end{array}$ & $\begin{array}{l}\text { Demanda } \\
\text { Física }\end{array}$ & $\begin{array}{l}\text { Demanda } \\
\text { Temporal }\end{array}$ & Performance & Esforço & $\begin{array}{c}\text { Nível de } \\
\text { Frustação }\end{array}$ & Soma \\
\hline 1 & 13,5 & 3 & 13,5 & 13,5 & 12 & 14 & 55,5 \\
\hline 2 & 13 & 2 & 10,5 & 12 & 9 & 6 & 52,5 \\
\hline 3 & 14 & 1 & 11 & 10 & 10,5 & 7 & 53,5 \\
\hline 4 & 14 & 7 & 15 & 15 & 7 & 3 & 61 \\
\hline 5 & 15 & 4 & 10,5 & 11 & 9 & 4 & 53,5 \\
\hline 6 & 11 & 8 & 10 & 11 & 13,5 & 14 & 67,5 \\
\hline 7 & 14 & 4 & 7 & 11 & 11 & 11 & 58 \\
\hline 8 & 6 & 4 & 10 & 7,5 & 7,5 & 0 & 35 \\
\hline 9 & 14 & 13 & 13,5 & 14 & 13,5 & 14 & 82 \\
\hline 10 & 9 & 7 & 11 & 13 & 10,5 & 14 & 64,5 \\
\hline 11 & 13 & 5 & 15 & 13,5 & 13 & 3 & 62,5 \\
\hline 12 & 11 & 5,5 & 13 & 13,5 & 10 & 7 & 60 \\
\hline 13 & 15 & 2 & 12 & 13,5 & 13,5 & 14 & 70 \\
\hline 14 & 13,5 & 13,5 & 13,5 & 10,5 & 11 & 2 & 64 \\
\hline 15 & 12 & 5 & 13,5 & 8 & 13 & 12 & 63,5 \\
\hline 16 & 10 & 3 & 14 & 13 & 10,5 & 10 & 60,5 \\
\hline 17 & 15 & 2 & 14 & 12 & 12 & 14 & 69 \\
\hline 18 & 15 & 7,5 & 9 & 11 & 8 & 13,5 & 64 \\
\hline 19 & 12 & 9 & 11 & 13 & 14 & 2 & 61 \\
\hline 20 & 13,5 & 10,5 & 15 & 13,5 & 13,5 & 12 & 78 \\
\hline 21 & 13,5 & 1 & 6 & 10,5 & 9 & 7,5 & 47,5 \\
\hline 22 & 14 & 13,5 & 13,5 & 10 & 14 & 12 & 77 \\
\hline 23 & 13,5 & 9 & 11 & 13 & 14 & 8 & 68,5 \\
\hline 24 & 13 & 5 & 15 & 13,5 & 13 & 3 & 62,5 \\
\hline 25 & 13 & 1 & 6 & 15 & 7,5 & 10 & 52,5 \\
\hline 26 & 15 & 2 & 13,5 & 12 & 10 & 2 & 54,5 \\
\hline 27 & 13 & 6 & 13 & 13 & 13 & 14 & 72 \\
\hline 28 & 12 & 3 & 13,5 & 13 & 13,5 & 12 & 67 \\
\hline 29 & 15 & 12 & 15 & 13 & 13 & 4 & 72 \\
\hline 30 & 11 & 3 & 10,5 & 13,5 & 12 & 7,5 & 57,5 \\
\hline 31 & 13,5 & 10 & 12 & 15 & 13,5 & 15 & 79 \\
\hline 32 & 13,5 & 9 & 13,5 & 13,5 & 14 & 12 & 75,5 \\
\hline 33 & 14 & 3 & 15 & 9 & 9 & 13 & 63 \\
\hline
\end{tabular}

Tabela 4. Carga de Trabalho de respondente.

\begin{tabular}{cccc}
\hline Respondente & CT Total & Respondente & CT Total \\
\hline 1 & 13,27 & 18 & 12,30 \\
2 & 11,23 & 19 & 12,33 \\
3 & 11,07 & 20 & 13,60 \\
4 & 12,07 & 21 & 10,30 \\
5 & 11,23 & 22 & 13,27 \\
6 & 12,60 & 23 & 12,27 \\
7 & 11,33 & 24 & 13,13 \\
8 & 7,20 & 25 & 11,50 \\
9 & 13,83 & 26 & 12,37 \\
10 & 12,30 & 27 & 13,33 \\
11 & 13,13 & 28 & 12,97 \\
12 & 11,57 & 29 & 14,13 \\
13 & 13,53 & 30 & 11,60 \\
14 & 12,60 & 31 & 14,30 \\
15 & 12,47 & 32 & 13,20 \\
16 & 12,23 & 33 & 13,07 \\
\hline
\end{tabular}


que não, observa-se que os alunos que trabalham têm uma demanda temporal e um esforço maior do que os que não trabalham e uma demanda mental menor. Além disso, o nível de frustração dos que trabalham é ligeiramente maior. Os valores são apresentados na Tabela 6.

Nas entrevistas, observou-se que ambas as categorias do mestrado relatam dificuldade com a demanda temporal, sendo que o mestrando profissional afirmou que

[...] o mestrado profissional pressupõe que o aluno trabalha e quer aplicar na sua organização o aprendizado acadêmico, mas com a demanda de atividades domiciliares, torna-se inviável esta aplicação, além de dificultar a entrega do trabalho na data imposta $[. .$.$] .$

Esse entrevistado ainda relatou que

[...] o mais difícil foi o primeiro semestre, onde conciliar a vida de demandas exigentes no trabalho com a vida acadêmica também exigente... foi tão árduo que para o segundo semestre optei por me desligar do vínculo empregatício e me dedicar exclusivamente ao mestrado profissional [...].

Já, segundo o relato do mestrando acadêmico,

[...] pelo fato de ser mestrando acadêmico, as cobranças são intensas, e para que seja feito um trabalho de qualidade, objetivando contribuições relevantes, não há tempo suficiente disponível, devido à grande demanda de atividades domiciliares [...],

o que vem ao encontro do analisado por meio do NASA TLX adaptado.

Tabela 5. Carga de trabalho média por categoria de respondente.

\begin{tabular}{ccc}
\hline & CT média geral & $\mathbf{1 2 , 4 0}$ \\
\hline Nível & Doutorado & 11,54 \\
de Pós- & Mestrado Acadêmico & 12,51 \\
Graduação & Mestrado Profissional & 13,23 \\
Sexo & Feminino & 12,33 \\
& Masculino & 12,46 \\
Trabalha? & Não & 12,23 \\
& Sim & 12,49 \\
\hline
\end{tabular}

Em linha com os resultados do NASA-TLX, o relato do doutorando destacou a demanda mental, como segue:

[...] uma dificuldade encontrada foi com relação à estrutura, desenvolvimento e publicação das pesquisas... ou seja, precisei me flexibilizar aos pensamentos diferentes, estruturas diferentes, formatos de pesquisa diferentes, necessitando de uma concentração mental enorme [...].

Na reunião de feedback dos resultados, os relatos acima foram entendidos como representativos pelos participantes, os quais também apontaram fatores contribuintes para a carga de trabalho, quais sejam: falta de acompanhamento do orientador, dificuldade na gestão de tempo, mudanças frequentes nas demandas dos professores, realização de trabalhos acadêmicos não diretamente ligados ao projeto de pesquisa como participação em atividades de extensão ou projetos de pesquisa institucionais, cobrança excessiva feita pelos estudantes a si mesmos, medo de não finalizar o trabalho, demandas domiciliares. A variedade de fatores implica a necessidade de ações de controle também variadas, passando pelo nível individual de cada estudante, por ações do programa de pós-graduação e da instituição como um todo, visto que pós-graduandos de outras áreas podem estar enfrentando dificuldades similares.

\section{Conclusões}

O presente artigo teve como objetivo contribuir com o estudo da carga de trabalho de alunos de pós-graduação. Para tanto, o questionário NASA-TLX adaptado foi aplicado a 33 alunos de um curso de pós-graduação em engenharia da produção, complementado por três entrevistas individuais e uma reunião de feedback dos resultados. A análise dos dados do questionário foi realizada segundo categorias como a modalidade de pós-graduação dos respondentes e o fato de realizar outros trabalhos além das atividades acadêmicas ou não.

Os resultados do questionário indicaram muita similaridade na carga de trabalho percebida entre as diferentes categorias (tipo de curso, sexo e trabalho), sendo que a maior diferença entre as médias gerais

Tabela 6. Valores individuais dos fatores.

\begin{tabular}{ccccccc}
\hline & Geral & Doutorado & $\begin{array}{c}\text { Mestrado } \\
\text { Acadêmico }\end{array}$ & $\begin{array}{c}\text { Mestrado } \\
\text { Profissional }\end{array}$ & Trabalha & $\begin{array}{c}\text { Não } \\
\text { Trabalha }\end{array}$ \\
\hline Demanda Mental & 2,48 & 2,91 & 2,43 & 2,03 & 2,10 & 3,13 \\
Demanda Física & 0,16 & 0,03 & 0,25 & 0,11 & 0,21 & 0,08 \\
Demanda Temporal & 2,69 & 1,91 & 2,96 & 3,05 & 3,17 & 1,86 \\
Performance & 2,58 & 2,42 & 2,57 & 2,80 & 2,48 & 2,74 \\
Esforço & 2,47 & 2,24 & 2,61 & 2,42 & 2,65 & 2,15 \\
Nível de Frustração & 2,03 & 2,02 & 1,70 & 2,82 & 1,89 & 2,28 \\
\hline
\end{tabular}


ocorreu entre a carga de trabalho do doutorado e a do mestrado profissional, sendo essa última um pouco maior $(1,69)$. Contudo, a carga de trabalho está em um nível de máximo risco para todas as categorias pesquisadas.

Já na análise individual dos fatores que contribuem para a carga de trabalho, destacam-se: (i) a inexistência de demanda física em todas as categorias; (ii) o alto nível de frustração do mestrado profissional; (iii) o alto nível de esforço do mestrado acadêmico; (iv) o alto nível de demanda mental do doutorado; (v) a maior demanda temporal de quem trabalha; (vi) a maior demanda mental de quem não trabalha.

Dentre as limitações deste trabalho, destacam-se a restrição da amostra a um curso de pós-graduação e o período de realização da pesquisa. Outra limitação diz respeito a não terem sido coletados dados mais precisos acerca do quanto o respondente de fato tinha avançado na sua pesquisa, nem de quais eram suas principais atividades no período da coleta de dados. De fato, tais informações poderiam ser úteis, visto que a carga de trabalho possivelmente tem picos e vales em determinados momentos da pós-graduação.

Em decorrência dessa pesquisa, algumas oportunidades de estudos futuros foram identificadas, tais como: (i) realizar estudos similares, em outros cursos de pós-graduação em engenharia de produção brasileiros, permitindo a comparação de resultados e a obtenção de conclusões mais generalizáveis - em tais estudos, sugere-se que a caracterização dos respondentes acrescente novas variáveis, como os horários de estudos extraclasse e o grau de avanço na pesquisa e atividades em desenvolvimento; (ii) realizar avaliações da carga de trabalho dos professores, visto que esta pode estar relacionada à carga dos estudantes; (iii) investigar em maior profundidade a natureza dos fatores contribuintes da carga de trabalho e suas inter-relações; e (iv) identificar e testar o impacto de ações de controle da carga de trabalho.

\section{Referências}

Adamo, S. A. (2013). Attrition of women in the biological sciences: workload, motherhood, and other explanations Revisited. Bioscience, 63(1), 43-48.

Adebayo, D. O. (2006). Workload, social support, and work-school conflict among nigerian nontraditional students. Journal of Career Development, 33(2), 125141. http://dx.doi.org/10.1177/0894845306289674.

Al-Sowygh, Z. H. (2013). Academic distress, perceived stress and coping strategies among dental students in Saudi Arabia. Saudi Arabia. The Saudi Dental Journal, 25(3), 97-105. http://dx.doi.org/10.1016/j.sdentj.2013.05.002. PMid:24179318.

Balch, C. M., Shanafelt, T. D., Dyrbye, L., Sloan, J. A., Russell, T. R., Bechamps, G. J., \& Freischlag, J. A. (2010). Surgeon distress as calibrated by hours worked and nights on call. American College of Surgeons, 211(5), 609-619. http://dx.doi.org/10.1016/j.jamcollsurg.2010.06.393. PMid:20851643.

Cardoso, M., \& Gontijo, L. (2012). Avaliação da carga mental de trabalho e do desempenho de medidas de mensuração: NASA TLX e SWAT. Gestão \& Produção, 19(4), 873-884.

Darmody, M., Smyth, E., \& Unger, M. (2008). Field of study and students' workload in higher education. International Journal of Comparative Sociology, 49(4-5), 329-346. http://dx.doi.org/10.1177/0020715208093080.

Diniz, R. L. (2003). Avaliação das demandas físicas e mental no trabalho do cirurgião em procedimentos eletivos (Tese de doutorado). Universidade Federal do Rio Grande do Sul, Porto Alegre.

Erichsen, E. A., \& Bolliger, D. U. (2011). Towards understanding international graduate student isolation in traditional and online environments. Educational Technology Research and Development, 59(3), 309-326. http://dx.doi.org/10.1007/s11423-010-9161-6.

Geslani, G. P., \& Gaebelein, C. J. (2013). Perceived stress, stressors, and mental distress among doctor of pharmacy sutudents. Social Behavior and Personality, 41(9), $1457-$ 1468. http://dx.doi.org/10.2224/sbp.2013.41.9.1457.

Grady, R. K., La Touche, R., Oslawski-Lopez, J., Powers, A., \& Simacek, K. (2014). Betwixt and Between: The Social Position and Stress Experiences of Graduate Students. Teaching Sociology, 42(1), 5-16. http://dx.doi. org/10.1177/0092055X13502182.

Graybill, J. K., Dooling, S., Shandas, V., Withey, J., Greve, A., \& Simon, G. L. (2006). A rough guide to interdisciplinarity: graduate student perspectives. Bioscience, 56(9), 757. http://dx.doi.org/10.1641/00063568(2006)56[757:ARGTIG]2.0.CO;2.

Gregoriades, A., \& Sutcliffe, A. (2008). Workload prediction for improved design and reliability of complex systems. Reliability Engineering \& System Safety, 93(4), 530-549. http://dx.doi.org/10.1016/j.ress.2007.02.001.

Hart, S. G., \& Staveland, L. E. (1988). Development of NASA-TLX (Task Load Index): results of empirical and theoretical research. In P. A. Hancock \& N. Meshkati (Eds.), Human mental workload. Amsterdam: North Holland Press.

Holsbach, L. (2005). Análise dos fatores humanos $e$ organizacionais nos incidentes em anestesia (Tese de doutorado). Universidade Federal do Rio Grande do Sul, Porto Alegre.

Instituto Brasileiro de Geografia e Estatística - IBGE. (2004). Censo Demográfico 2000: educação. Rio de Janeiro: Ministério do Planejamento, Orçamento e Gestão.

Instituto Brasileiro de Geografia e Estatística - IBGE. (2012). Censo Demográfico 2010: educação. Rio de Janeiro: Ministério do Planejamento, Orçamento e Gestão. 
Jacobs, S. R., \& Dodd, D. (2003). Student burnout as a function of personality, social support, and workload. Journal of College Student Development, 44(3), 291303. http://dx.doi.org/10.1353/csd.2003.0028.

Johnson, C., \& Smith, E. C. (2008). A mid-term terminal concept of operations: evaluation and evolution analysis. Bethesda: IEEE.

Kyndt, E., Dochy, F., Struyven, K., \& Cascallar, E. (2011). The direct and indirect effect of motivation for learning on students' approaches to learning through the perceptions of workload and task complexity. Higher Education Research \& Development, 30(2), 135-150.

Lewis, J. S., \& Webb, T. (2009). Student assignment workload: students' perceptions compared to lecturers' expectations. In: 20th Annual Conference for the Australasian Association for Engineering Education. Barton: Engineers Australia.

Meshkati, N., Hancock, P. A., \& Rahimi, M. (1992). Techniques in mental workload assessment. In J.
Wilson \& E. Corlett (Eds.), Evaluation of human work: a practical ergonomics methodology (pp. 605-627). London: Taylor \& Francis.

Myrtek, M., Weber, D., Brügner, G., \& Müller, W. (1996). Occupational stress and strain of female students: results of physiological, behavioral, and psychological monitoring. Biological Psychology, 42(3), 379-391. http://dx.doi. org/10.1016/0301-0511(95)05168-6. PMid:8652754.

Panari, C., Guglielmi, D., Ricci, A., Tabanelli, M. C., \& Violante, F. S. (2012). Assessing and improving health in the workplace: an integration of subjective and objective measures with the STress Assessment and Research Toolkit (St.A.R.T.) method. Journal of Occupational Medicine and Toxicology, 7(1), 18. http:// dx.doi.org/10.1186/1745-6673-7-18. PMid:22995286.

Stoll, R., Kreuzfeld, S., Weippert, M., Vilbrandt, R., \& Stoll, N. (2007). System for flexible field measurement of physiological data of operators working in automated labs. Journal of Laboratory Automation, 12(2), 110-114. 
Anexo 1. Questionário NASA-TLX.

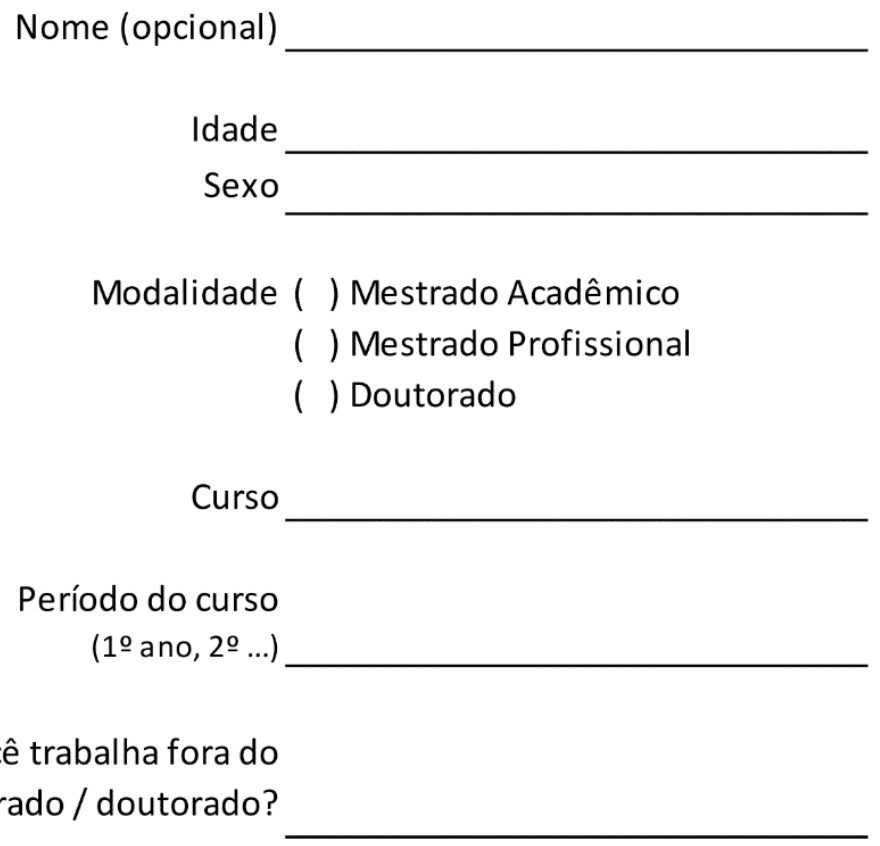

\section{Com base nesses conceitos, responda as perguntas a seguir}

Demanda Mental - atividade mental requerida para realização de um trabalho

Demanda Física - atividade física requerida para a realização de um trabalho

Demanda Temporal - nível de pressão imposta para a realização de um trabalho

Performance - Nível de satisfação com desempenho pessoal para a realização de um trabalho

Esforço - O quanto que se tem que trabalhar fisica e mentalmente para atingir um nível desejado de performance ou desempenho

Nível de Frustração - Nível de fatores que inibem a realização do trabalho (insegurança, irritação, falta de estímulo, estresse, contrariedades). 
Marque " $X$ " em um dos fatores, entre os pares abaixos, que você considera como a fonte mais significativa para a carga de trabalho durante a realização de suas tarefas de mestrado
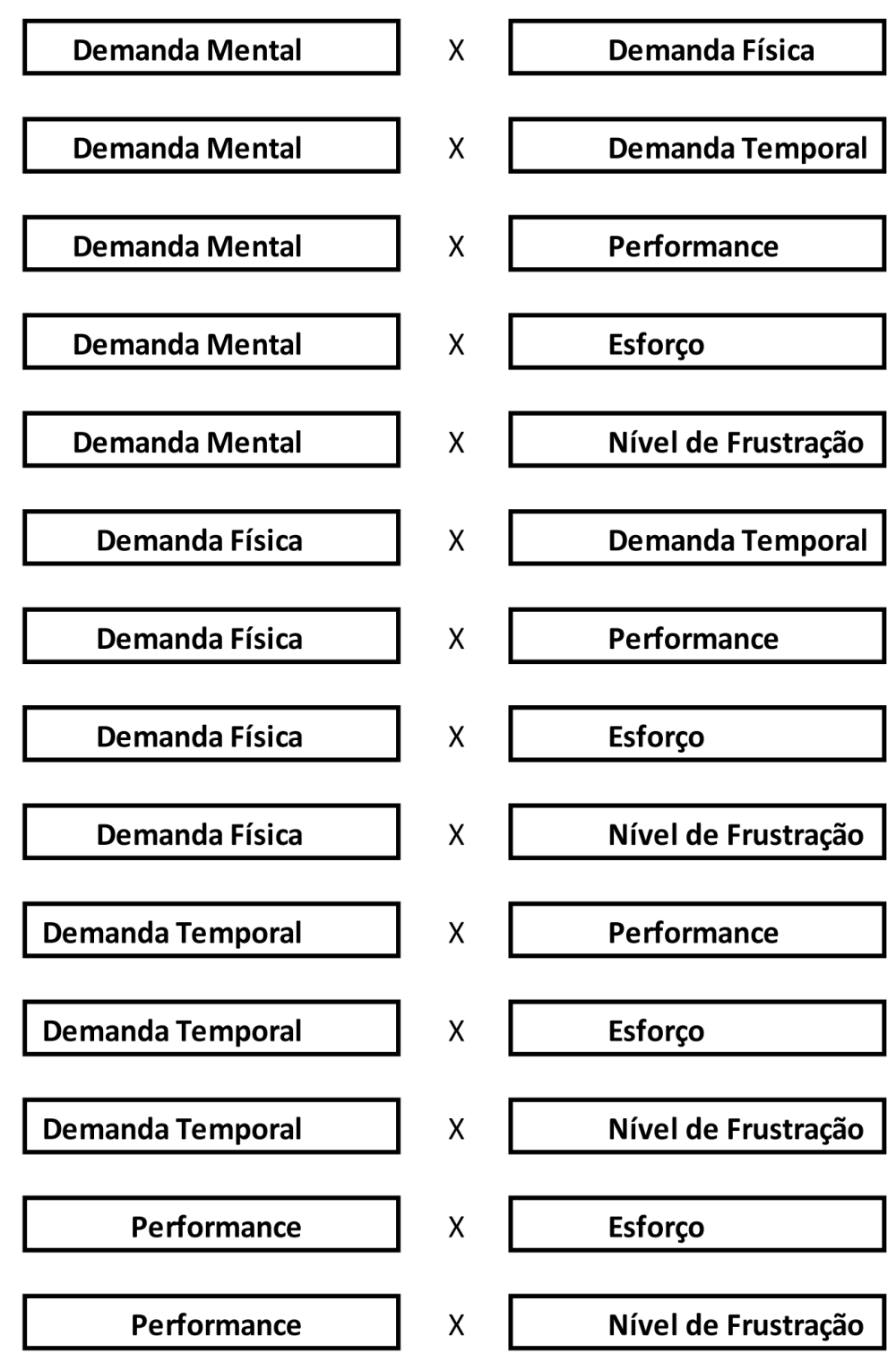

Nível de Frustração

$\mathrm{X}$

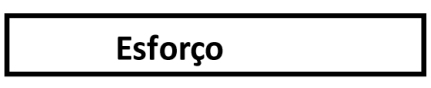

EXEMPLO

Demanda Mental

$X \quad X \quad$ Demanda Física 
Marque na escala qual a sua opinião sobre o nivel de influência dos fatores abaixo para a realização do seu mestrado

EXEMPLO

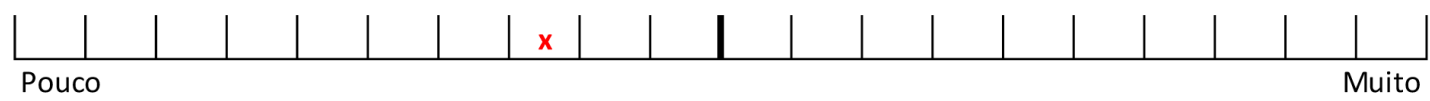

Demanda Mental

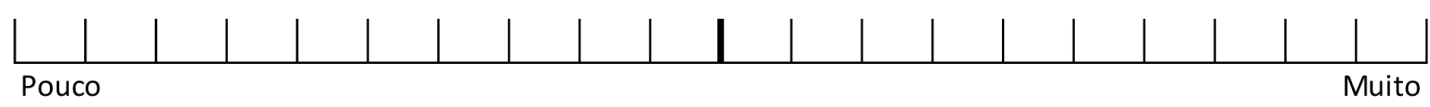

Demanda Física

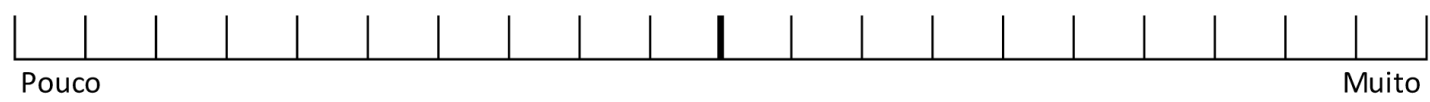

Demanda Temporal

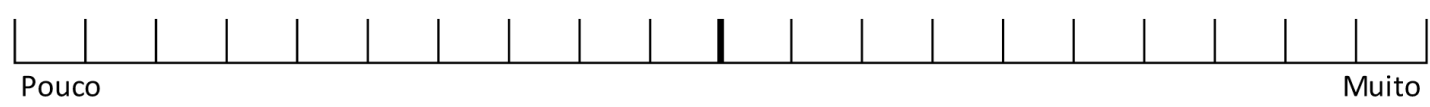

Performance

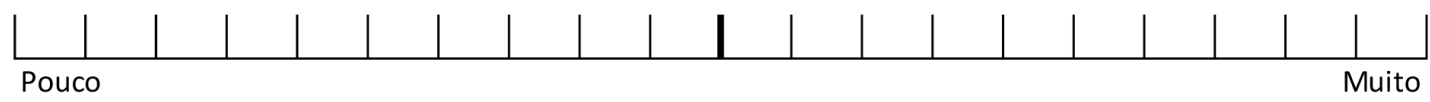

Esforço (Físico e Mental)

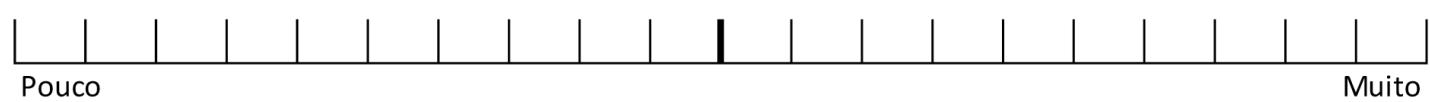

Nível de Frustação

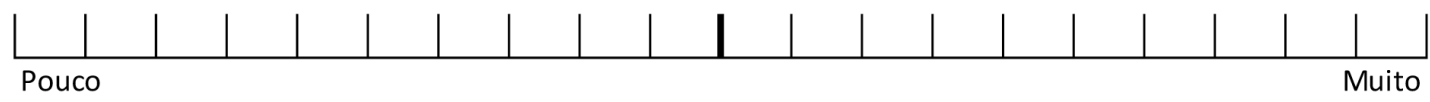

